\title{
SOARING MOTION OF VIBRATING DRIVE OBJECT IN FLUID
}

\author{
Edgars Kovals, Maris Eiduks, Kristaps Spade, Janis Viba, Kaspars Burbeckis \\ Riga Technical University, Latvia \\ edgars.kovals@gmail.com,maris.eiduks@rtu.lv, kristaps.spade@rtu.lv, janis.viba@rtu.lv, \\ kaspars.burbeckis@temeso.lv
}

\begin{abstract}
The authors' research on the application of vibrating motors in technology, technologies and everyday life is continued. Unlike previous studies on the movement of an object over the dry surface of another object, the movement in fluid (water, air) is analyzed here. The movement in the vertical plane of the object, which on the outside consists of a monolithic body with wide wings, is considered. Inside this first object is a vibrator or a second moving object controlled by mechatronics. A simplified movement of an object is considered, in which the rotation around the center of mass does not take place, which imposes additional rules for the synthesis of the system design. The motion is described by two second-order differential equations, which take into account: gravity interaction; - buoyancy or the Archimedes' principle; - the forces of interaction between the hull and the fluid, which depend on the square of the absolute speed of the translational movement of the hull; - similar wing interaction force; - law of relative motion control of vibrator; In addition, the possibility of mechatronic motion control by changing the angle of the wings towards the body has been used. The obtained differential equations are analyzed numerically at different internal vibrator motion laws. In addition, the soaring motion of the object, which depends on the control of the wing angle, as well as the additional motion of the fluid flow, have been studied. The research results are illustrated with phase coordinate graphs. The results obtained in the work can be used for the analysis, optimization and synthesis of new flying and soaring objects.
\end{abstract}

Keywords: fluid-rigid body interaction, soaring motion.

\section{Introduction}

In technics, technological processes and nature, people often encounter soaring objects. It takes place under water, on the surface of the water and in the air. For example, under the water there is gliding of fish and also slip of the submarine, a water skier or a wake board moves on the surface of the water, but birds and airplanes fly (Fig. 1-3).

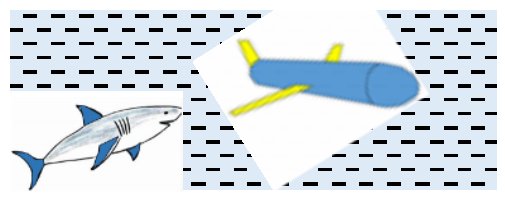

Fig. 1. Gliding of objects under water

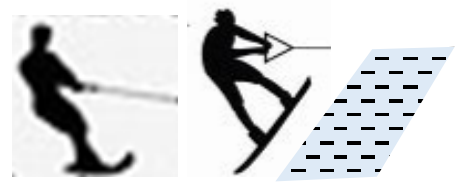

Fig. 2. Movement of objects on the water surface

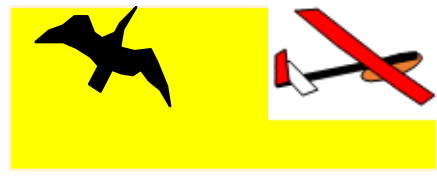

Fig. 3. Gliding of objects in the air

When analyzing the different movements of these objects, it is important to note that their interaction with fluid is very complex. The main problem is that both - the object itself and the interaction fluid - are in motion. In this way, a mechanical system with an infinite number of degrees of freedom must be studied, using approximate analytical or numerical methods. It should be noted here that the usual formula for describing fluid dynamics with drag and lift coefficients is well applicable in cases of stationary motion [1-3], where speeds of movement and areas of interaction are constant over time. However, in cases of non-stationary movements, such as when a bird flaps its wings or other parameters of the interaction change, a work-intensive space-time calculation program should be used [4]. Our work will show how to apply an indicative analytical theory using the formulas of classical mechanics [5-6]. As for the shapes and parameters of different soaring objects, they must be considered separately and analyzed in the calculations. For example, some important questions about aircraft parameters and bird wing parameters are addressed in articles [7-13]. Accordingly, the analysis and synthesis of real motion of flying objects can be found, for example, in articles $[14 ; 15]$. Various studies on the kinematics of insects in terms of flight efficiency and optimization issues can be found, for example, in [16].

These studies can be used in micro air vehicles, where low energy consumption is required at relatively high power output.Here, our further analysis will look at some of the main cases of object soaring motion. This will make it possible to understand and apply the proposed analytical method in the analysis of the motion of these special objects. 


\section{Materials and methods}

\section{Model and analysis method for movement in a vertical plane}

We start the study of the motion in a fluid with a simple movement, when the object moves vertically upwards (Fig. 4).

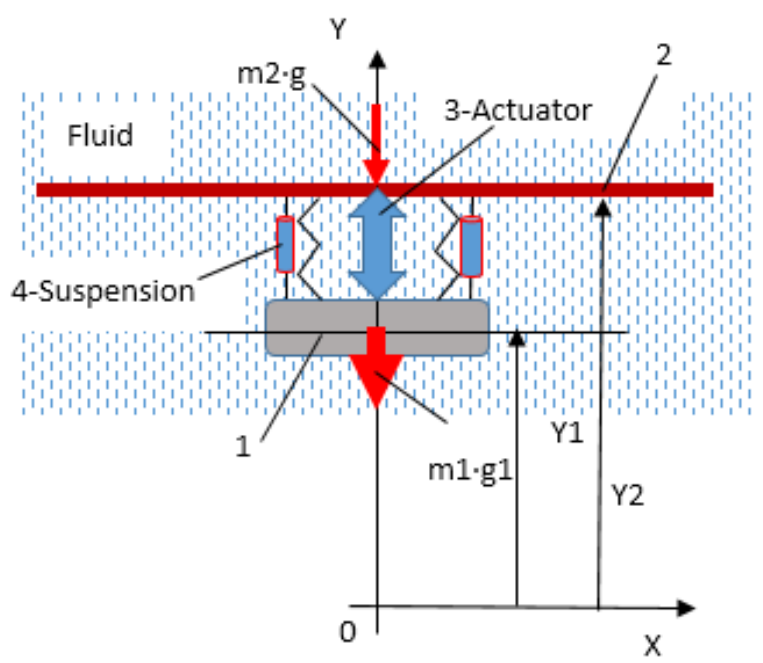

Fig. 4. Model of movement in the vertical plane: 1 - central mass; 2 - movable flat plate;

3 - internally driven actuator; 4 - internal flexible suspension

The differential equations of motion of a given system of two degrees of freedom (2DOF) in a fixed reference system are as follows (1), (2 [5]):

$$
\begin{aligned}
m 1 \cdot \ddot{y} 1 & =A 1-m 1 \cdot g-b 1 \cdot(\dot{y} 1)^{2} \cdot \operatorname{sign}(\dot{y} 1)-[c 12 \cdot(y 1-y 2)+b 12 \cdot(\dot{y} 1-\dot{y} 2)]-Q ; \\
m 2 \cdot \ddot{y} 2 & =A 2-m 2 \cdot g-\{[(1+C) \cdot \rho \cdot L \cdot B] \cdot(1-k \cdot \operatorname{sign}(\dot{y} 2))\} \cdot(\dot{y} 2)^{2} \cdot \operatorname{sign}(\dot{y} 2)+ \\
& +[c 12 \cdot(y 1-y 2)+b 12 \cdot(\dot{y} 1-\dot{y} 2)]+Q),
\end{aligned}
$$

were $\quad m 1, m 2-$ mases of bodies 1 and 2 ;

$\ddot{y} 1, \ddot{y} 2, \dot{y} 1, \dot{y} 2, y 1, y 2$ - accelerations, velocities and displacement of bodies;

$A 1, A 2$ - forces of Archimedes' principle;

$g$ - free fall acceleration;

$\rho$ - fluid density;

$L, B$ - length and width of the thin plate;

$Q$ - internal excitation force;

$b 1, b 2, b 12$ - external and internal damping coefficients;

$c 12$ - linear spring stiffness;

$k$ - plate area exchange constant.

Accordingly, for long plate $C=0.5[6 ; 7]$. It is important to note the fact that the differential equation of the mechatronic system (2) parameter $\mathrm{k}$ switches the interaction area. It roughly imitates the bird's wings.

The movement of the vertical motion in the fluid was simulated $(A 1=\sim 0 ; A 2=\sim 0)$ at the following parameters (System SI): $m 1=0.2 ; \quad m 2=0.05 ; g=9.81 ; \quad c 12=2000 ; \quad b 1=2.5 \cdot 10^{-3} ; \quad b 2=0.25$; $b 12=0.0001 ; k=0.5$.

Accordingly, the actuator interaction $\mathrm{Q}$ was adopted as biharmonic, in the form:

$$
Q=\Delta \cdot[\sin (\omega \cdot t)+\lambda \cdot \sin (2 \cdot \omega \cdot t+\varphi)],
$$

where $\Delta=98.1 ; \omega=314 ; \lambda=1 ; \varphi=0,5 \cdot \pi ; t$ - time. 
Some modeling results are shown in Fig. (2-5), where movement starts from the rest position, accordingly, that is: $\dot{x} 1(0)=0 ; \quad \dot{x} 2(0)=0$.

Here it is interesting to note the fact that at the beginning the mass $m 1$ is falling down, but further begins ascending. Of course, as it is understandable, flight to the top is not possible with all parameters. It depends on $m 1$ and $m 2$ relations (big or small).

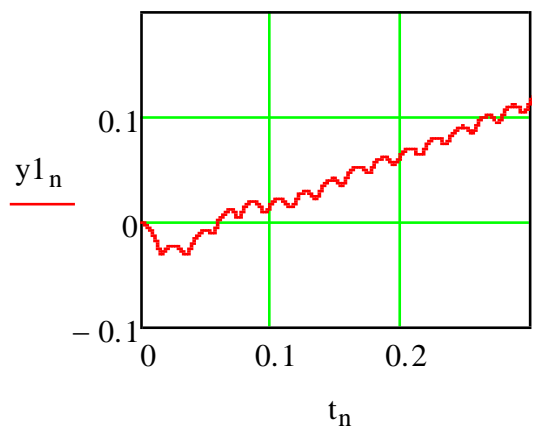

Fig. 5. Mass $m 1$ vertical movement as a function of time

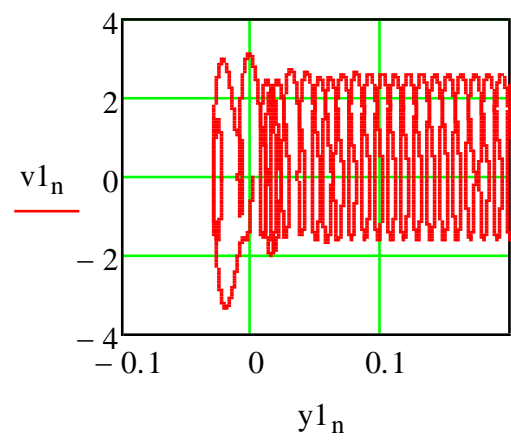

Fig. 7. Mass $m 1$ motion illustration in phase plane

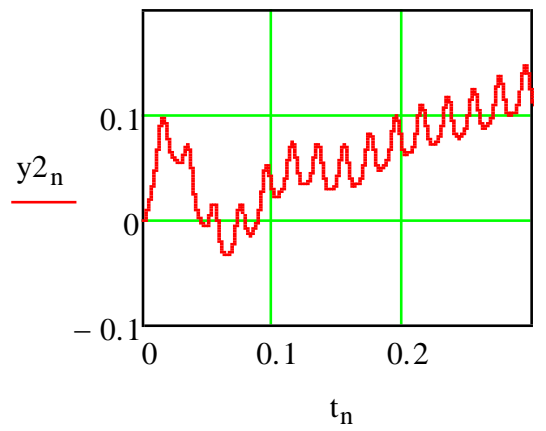

Fig. 6. Mass $m 2$ vertical movement as a function of time

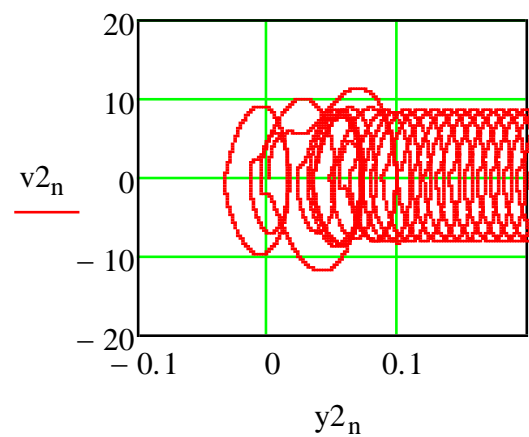

Fig. 8. Mass $m 2$ motion illustration in phase plane

\section{Mathematic model of plane soaring movement in a vertical plane}

Model of plane soaring movement is shown in Fig. 9. Consider the case where the internal actuator 3 (Fig. 1) kinematically provides the relative displacement

$$
\mathbf{r} 2=\left\{\frac{x}{y}\right\},
$$

given to the mass $\mathrm{m} 2$ against the mass $\mathrm{m} 1$. In the moving reference system $\mathrm{xC} 1 \mathrm{y}$ (Fig. 9), the electromechanical system then again has two degrees of freedom of movement (2DOF), which are described by the following two differential equations (3) in vector form [5]:

$$
\begin{aligned}
(m 1+m 2) \cdot\left\{\frac{\ddot{X} 2}{\ddot{Y} 2}\right\} & =-m 1 \cdot\left\{\frac{\ddot{x}}{\ddot{y}}\right\} \cdot\left[\begin{array}{cc}
\cos (\varphi) & -\sin (\varphi) \\
\sin (\varphi) & \cos (\varphi)
\end{array}\right]+ \\
& +(A 1+A 2)\left\{\frac{0}{1}\right\}-(m 1+m 2) \cdot g \cdot\left\{\frac{0}{1}\right\}+N \cdot\left\{\frac{-\sin (\varphi)}{\cos (\varphi)}\right\} .
\end{aligned}
$$

where $\left\{\begin{array}{l}\ldots \\ \ldots\end{array}\right\}$ is a two-dimensional vector, and $\left[\begin{array}{ll}\cdot & \cdot \\ \cdot & .\end{array}\right]$ is a matrix. 
Here $N$ is the normal force of the interaction between the body $\mathrm{m} 2$ and the fluid, directed perpendicular to the soaring plane $\mathrm{x}$ and equals (4):

$$
N=\rho \cdot L \cdot B \cdot C 0 \cdot\left(V_{N}\right)^{2} \cdot \operatorname{sign}\left(V_{N}\right),
$$

where for a symmetrical triangular prism with the wing angle $\beta$ (Fig. 9) the coefficent $\mathrm{C} 0$ equals

$$
C 0=\left[\cos \left(\beta \cdot\left(0.5+0.5 \cdot \operatorname{sign}\left(V_{N}\right)\right)\right]^{2}+C,\right.
$$

and $V_{N}$ is a fluid relative velocity projection to plate normal (6) (Fig. 10.)

$$
V_{N}=V 0 \cdot \sin (\gamma-\varphi)-\dot{X} 2 \cdot \sin (\varphi)-\dot{Y} 2 \cdot \cos (\varphi),
$$

where $v 0$ - velocity of the wind flow acting on the mass $m 2$ at an angle $\gamma$ to the horizon.

Thus, the description of the soaring motion of a 2DOF mechanical system must integrate two vector equations (3) at a given initial conditions.

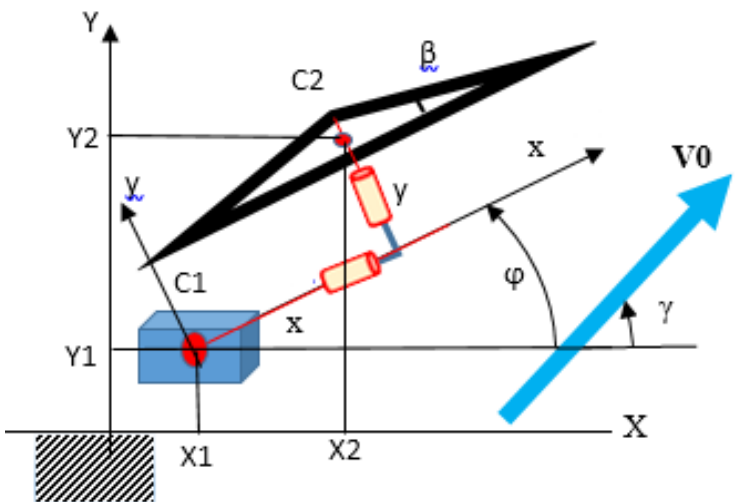

Fig. 9. Plane soaring model

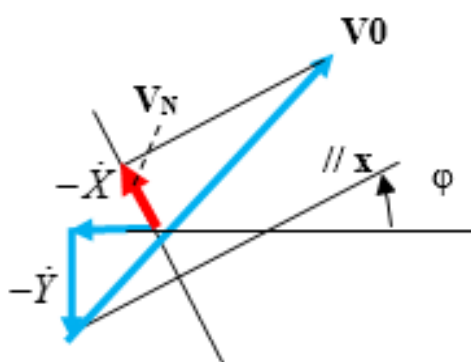

Fig. 10. Calculation of a fluid relative velocity $V_{N}$.

\section{Object motion analysis with computer modellingUncontrolled soaring movement of a triangular prism}

The obtained systems of equations were used to validate the application of the following flapping and soaring object motion models:

- passive flat wing object soaring with the angle $\varphi=-\pi \cdot 10^{-1}$ in the vertical plane (Fig. 11);

- soaring of a flat wing object at the steering angle $\varphi=-\pi \cdot 10^{-1} \cdot\left(1+\sin \left(\pi \cdot 10^{-1} \cdot \mathrm{t}\right)\right)$ in a vertical plane (Fig. 12);

- (c) soaring flight of a controlled object by means of wing vibrations (Fig. 13).

a)

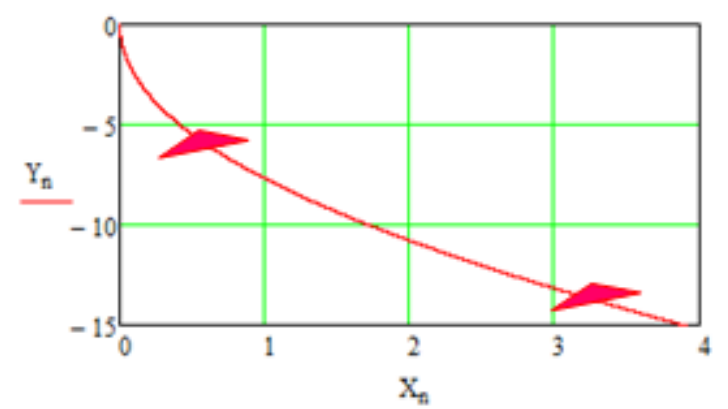

b)

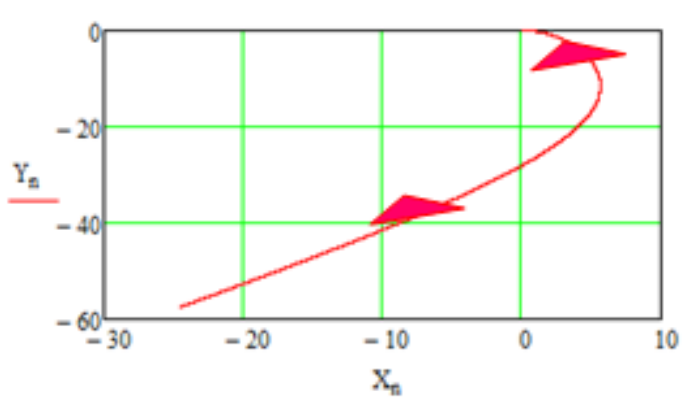

Fig. 11. Trajectory of passive flat wing object soaring: $\mathrm{a}$ - motion from rest position; $\mathrm{b}-$ motion with given velocity ahead, then soaring back as boomerang. 


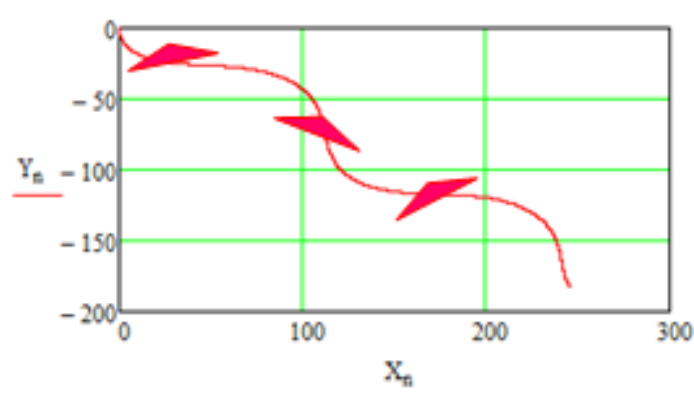

Fig. 12. Soaring of a flat wing object at harmonica controlled angle: $\varphi=-\pi \cdot 10^{-1} \cdot\left(1+\sin \left(\pi \cdot 10^{-1} \cdot \mathrm{t}\right)\right)$

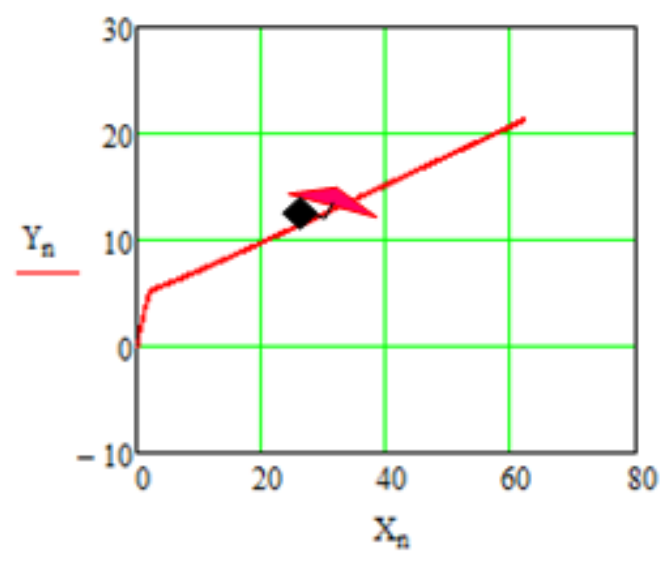

Fig. 13. Soaring flight of a controlled object by means of wing vibrations and wind action:

$\varphi=-\pi \cdot 10^{-1} ; x=0.05 \cdot \sin (100 \cdot \pi \cdot t+\pi / 2)$;

$y=0.1 \cdot \sin (100 \cdot \pi \cdot t+0) ; V 0=2 ; \gamma=0$

\section{Results and discussion}

The motion of a plate or triangle system can be described by an integral-differential equation, which is integrated and transformed into a normal nonlinear second-order differential equation. The obtained two equations can be numerically integrated at the given function of the given flow velocity. The proposed analytical method of calculation can be applied in the analysis of the movement of various soaring and flapping objects, including: the flight of insects or birds in the air; underwater object soaring motion analysis; studying the soaring motion of objects over the water surface. This article is a continuation of the latest works of the authors' team in fluid mechanics, where the motion of objects in still or moving fluid is studied [6; 7]. The work differs from previous studies by the moving object is not being connected to a fixed base but soaring in space. The work shows the advantages of the proposed new method over the conventional method of using drag coefficients. For example, the drag force method requires prior knowledge of the experimental drag and lift coefficients at each point in time.

The proposed method considers these coefficients during the motion from equations in which the projection of the relative velocity on the normal direction of the plane is analytically found. In this way, the given research offers a new method for studying practical tasks in technology, nature and everyday life, where it is possible to analytically describe the surface of the flying object with space - time equations. Examples of the application of the theory are the analysis and explanation of such movements as: bird (albatross, gull) gliding, boomerang flight, paper plane flight, analysis of the movement of new winged drones.

\section{Conclusions}

1. Classical mechanical formulas for the change of the moment of movement of a fluid interacting with a flat surface or triangle form are used to obtain the differential equation of motion of a flat soaring motion.

2. Ideas of soaring motion synthesis are offered, if the system parameters can be changed with mechatronic systems.

3. An approximate method of fluid interaction description is shown and used, which has its advantages in the synthesis of systems versus space-time numerical calculation methods.

\section{References}

[1] Clancy L.J., Aerodynamics. New York, London: Publishing by Pitman, 1975. 610. p.

[2] Sears W.R. Introduction to Theoretical Aerodynamics and Hydrodynamics, American Institute of Aeronautics and Astronautics, Reston, VA, 2011. 203. p. 
[3] Jiang H., Li Y., Cheng Z. Relations of Lift and Drag Coefficients of Flow around Flat Plate, Applied Mechanics and Materials, Vol. 518, 2014. pp 161-164.

[4] Tezduyar T.E., Sathe S. "Modeling of fluid-structure interactions with the space-time finite elements: Solution techniques", International Journal for Numerical Methods in Fluids, 54, 2007, pp. 855-900. [online] [21.03.2021] Available at: https://www.researchgate.net/publication/229866002

[5] Goldstein H., Poole C., Safko J. Classical Mechanics. Third Edition. 2015.

[6] Tipans I., Viba J., Irbe M., Vutukuru S.K. Analysis of Non-Stationary flow interaction with simple form objects. Agronomy Research. Vol. 17(1), 2019, pp. 1227-1234.

[7] Vutukuru S.K., Viba J., Tipans I., Viksne I., and Irbe M. Analysis of flat Plate Vibrations by varying frontal area to flow. Engineering for rural development. 2019, pp. 1408-1414.

[8] Kruyt J. W., Van Heijst G. F., Lentink D. I. Power reduction and the radial limit of stall delay in revolving wings of different aspect ratio. J. R. Soc. Interface 12: 20150051. DOI: 10.1098/rsif.2015.0051.

[9] Wang X., J. A Clarke. The evolution of avian wing shape and previously unrecognized trends in covert feathering. Proceedings of the Royal Society B, 2015 - royalsocietypublishing.org. DOI: $10.1098 / \mathrm{rspb} .2015 .1935$

[10] Harvey C., Baliga V.B., Lavoie P., Altshuler D. L. Wing morphing allows gulls to modulate static pitch stability during gliding. Journal of the Royal J. R. Soc. Interface, 2019 royalsocietypublishing.org. DOI: 10.1098/rsif.2018.0641.

[11] Baliga V. B., Szabo I., Altshuler D. L. Range of motion in the avian wing is strongly associated with flight behavior and body mass. Science Advances. 23 Oct. 2019:Vol. 5, no. 10, eaaw6670. DOI: $10.1126 /$ sciadv.aaw6670.

[12] Nesar Ali Md., Alam M., Abed Hossain Md., Imteaz Ahmed Md. Study on bird's \& insect's wing aerodynamics and comparison of its analytical value with standard airfoil. AIP Conference Proceedings 1851, 020056 (2017); DOI: 10.1063/1.4984685

[13] Williams H. J., Shepard E. L. C., Holton M.D., Alarcón P. A. E., Wilson R. P., Lambertucci S. A. Physical limits of flight performance in the heaviest soaring bird. PNAS July 28, 2020117 (30) pp. 17884-17890; DOI: 10.1073/pnas.1907360117

[14]Zhu L.L., Guan H., Wu C.J. A study of a three-dimensional self-propelled flying bird with flapping wings. Science China Physics, Mechanics \& Astronomy volume 58, Article number: 594701 (2015) DOI: $10.1007 / \mathrm{s} 11433-015-5686-3$

[15] Chin Y.-W., Kok J.M., Zhu Y.-Q., Chan W.-L., Chahl J.S., Khoo B.C., Lau G.-K. Efficient flapping wing drone arrests high-speed flight using post-stall soaring. Science Robotics 22 Jul 2020: Vol. 5 , Issue 44, eaba2386, DOI: 10.1126/scirobotics.aba2386.

[16] Hoang Vu Phan, Hoon Cheol Park. Design and evaluation of a deformable wing configuration for economical hovering flight of an insect-like tailless flying robot. Bioinspir Biomim2018 Apr 18;13(3):036009. doi: 10.1088/1748-3190/aab313. 